\title{
A Comparative Study of Oral Microbiota in Infants with Complete Cleft Lip and Palate or Cleft Soft Palate
}

\author{
Agnieszka Machorowska-Pieniążek, ${ }^{1}$ Anna Mertas, ${ }^{2}$ Małgorzata Skucha-Nowak, ${ }^{3}$ \\ Marta Tanasiewicz, ${ }^{3}$ and Tadeusz Morawiec ${ }^{4}$ \\ ${ }^{1}$ Department of Orthodontics, School of Medicine with the Division of Dentistry in Zabrze, Medical University of Silesia in Katowice, \\ Traugutta Square 2, 41-800 Zabrze, Poland \\ ${ }^{2}$ Department of Microbiology and Immunology, School of Medicine with the Division of Dentistry in Zabrze, \\ Medical University of Silesia in Katowice, Jordana 19, 41-808 Zabrze, Poland \\ ${ }^{3}$ Department of Conservative Dentistry with Endodontics, School of Medicine with the Division of Dentistry in Zabrze, \\ Medical University of Silesia in Katowice, Akademicki Square 17, 41-902 Bytom, Poland \\ ${ }^{4}$ Department of Dental Surgery, School of Medicine with the Division of Dentistry in Zabrze, Medical University of Silesia in Katowice, \\ Akademicki Square 17, 41-902 Bytom, Poland
}

Correspondence should be addressed to Agnieszka Machorowska-Pieniążek; agamach@onet.pl

Received 5 December 2016; Revised 12 February 2017; Accepted 7 March 2017; Published 14 March 2017

Academic Editor: Koichiro Wada

Copyright (C) 2017 Agnieszka Machorowska-Pieniążek et al. This is an open access article distributed under the Creative Commons Attribution License, which permits unrestricted use, distribution, and reproduction in any medium, provided the original work is properly cited.

Few reports have been published on the early microbiota in infants with various types of cleft palate. We assessed the formation of the oral microbiota in infants with complete cleft lip and palate (CLP $n=30)$ or cleft soft palate $(\operatorname{CSP} n=25)$ in the neonatal period ( $\mathrm{T} 1$ time) and again in the gum pad stage (T2 time). Culture swabs from the tongue, palate, and/or cleft margin at T1 and T2 were taken. We analysed the prevalence of the given bacterial species (the percentage) and the proportions in which the palate and tongue were colonised by each microorganism. At T1, Streptococcus mitis (S. mitis) were the most frequently detected in subjects with CLP or CSP (63\% and 60\%, resp.). A significantly higher frequency of methicillin-sensitive Staphylococcus aureus (S. aureus MSSA) was observed in CLP compared to the CSP group. At T2, significantly higher percentages of S. mitis, S. aureus MSSA, Staphylococcus epidermidis, and members of the Enterobacteriaceae family were noted in CLP infants compared to the CSP. S. mitis and Streptococcus sanguinis appeared with the greatest frequency on the tongue, whereas Streptococcus salivarius was predominant on the palate. The development of the microbiota in CLP subjects was characterised by a significant increase in the prevalence of pathogenic bacteria.

This paper is dedicated to the memory of colleague and friend Professor Wojciech Król, who recently died

\section{Introduction}

The oral cavity, which remains sterile throughout prenatal development, becomes a diverse ecosystem colonised by numerous microorganisms during the first hours following delivery. The skin and mucus membranes of neonates are colonised by microbiota as a result of contact with the external environment. A significant part of the oral microbiota in the early neonatal period originates from the mother and is transient population of microorganisms consisting of intestinal bacteria (in neonates born naturally) [1]. The resident microbiota in this period depends mainly on external factors, including gestational age, mode of delivery, type of feeding, the length of hospital stay following delivery, and general condition [1-10]. The complex structure of the oral cavity, with its numerous recesses, the mucosal folds of the palate, and the invaginations of the cheeks and tongue, creates niches with different $\mathrm{pH}$ values, local oxygen concentrations, redox 
states, ionic compositions, buffer capacities, hydration, access to saliva, and mechanical interactions. These conditions are favourable for the development of a diverse ecosystem based on the interactions between bacteria and the host environment [11, 12]. The early oral microbiota occurring within several hours following delivery is composed of viridans streptococci and Streptococcus salivarius (S. salivarius), which are commensals permanently colonising the oral cavity [2]. Along with other bacteria, they participate in the formation of a "colonisation cascade" that determines future indigenous microbiota $[2,5,6]$.

Congenital orofacial malformation affects the structure and functions of the oral cavity, thereby significantly modifying its characteristics [13]. As a result, such malformations may exert influence on the microbiota of the environment. Orofacial clefts are the most common congenital developmental malformation of the oral cavity [14]. Neonates with complete cleft lip and palate (CLP) are characterised by the existence of communication between oral and nasal cavities extending from the upper lip and nasal vestibule to the end of the soft palate. This condition adversely affects natural sucking or even impairs the ability to swallow food [15]. Moreover, neonates and infants with orofacial cleft require specialised care to maintain proper hygiene of the incisive bone, nasal passages, and the oral cavity with special attention paid to preparation for future surgical procedures [14]. Cleft soft palate (CSP) is a less severe form of orofacial cleft with the continuity of the lips and hard palate maintained. Dysmorphia of the oral cavity in patients with this malformation affects the dorsal part of the oral and nasal cavities, which are characterised by significantly reduced communication compared to CLP [16].

Previous studies have confirmed that patients with orofacial cleft are at increased risk for the development of caries and periodontal diseases compared to noncleft children [13, 14]. Furthermore, changes in the amount and composition of oral microbiota have been reported in subjects with different types of cleft palate during deciduous or permanent dentition [17] and as the result of surgical or orthodontic treatment [1820].

Both abnormal morphology and improper function of the oral cavity in newborns with cleft palate create a different environment from that of healthy neonates. Therefore, these abnormalities may affect oral microbiota [21]. Few reports have been published on the early microbiota in neonates and infants with various types of cleft palate.

The primary aim of the study was to compare the oral microbiota in infants with CLP and infants with CSP group. The second aim was to assess the development of the oral microbiota in subjects with complete CLP and age-matched CSP group during the neonatal period and then in the gum pad stage of the infancy period before surgery.

\section{Materials and Methods}

2.1. Design and Participants. This study was conducted from May 2012 to December 2014 in the Developmental Anomaly Outpatient Clinic at the Centre of Dentistry and Specialist Medicine, Medical University of Silesia in
Zabrze. The study was approved by the Bioethics Committee of the Medical University of Silesia in Katowice, Poland (KNW/0022/KB1/54/12). All legal guardians of the subjects enrolled in the study provided written consent for their participation.

The study materials consisted of microbiological smears from the oral cavity mucosa collected from neonates and infants with cleft malformation who were consulted and treated at the Developmental Anomaly Outpatient Clinic of the University Centre of Dentistry and Specialised Medicine in Zabrze, Poland.

The inclusion criteria for newborns were as follows: (1) complete CLP or CSP; (2) gestational age over 37 weeks, (3) birth weight of 2,500-4,000 g, and (4) Apgar score of 9-10 at $1 \mathrm{~min}$ and of 10 at $5 \mathrm{~min}$. The exclusion criteria were (1) the coexistence of orofacial cleft with other developmental abnormalities, (2) antibiotic therapy, (3) respiratory tract infections, (4) tube feeding, (5) treatment with palatal plate, (6) natal or neonatal teeth, (7) deciduous teeth at T2, (8) past surgical repair of cleft lip and/or palate, and (9) failure to appear for the follow-up visit between the eighth and eighteenth week of life. Figure 1 shows a flowchart of the process that was used to screen and select the trials.

At the first visit all parents were provided with the feeding instructions that were adapted individually to the needs of each patient. All mothers were encouraged to put the child to the breast. All patients with CLP were bottle-fed with a broad or standard nipple. Eight patients with CLP were fed partially with modified milk and partially with breast milk from the bottle. The remaining newborns/neonates were given modified milk only. Two patients with CSP were breastfed (within 3 and 6 weeks). After this period they were additionally fed with modified milk. Four neonates with CSP were bottle-fed (Haberman Feeder) with modified milk. The other patients were fed with the regular nipple and partially with modified milk and partially with breast milk from the bottle.

Feeding problems occurred in 16 patients (10 with CLP and 6 with CSP). The problems were related to the long feeding period (>40 min), choking, coughing, crying with feeding and regurgitation. In these patients a lower weight gain was observed within the first month of life by $~ 90-110 \mathrm{~g}$ per week.

The subjects were divided into two groups (Table 1). The first group consisted of 30 infants with unilateral or bilateral complete CLP. In this group, smears were obtained from palatal mucosa on the cleft margin (sample A1) and from the dorsum of the tongue (sample A2). The second group comprised 25 subjects with isolated CSP, and smears were obtained from the palatal mucosa (sample B1) and the dorsum of the tongue (sample B2). The samples were collected by rubbing the mucous membrane with a sterile cotton swab.

The smears were collected twice from the subjects of both groups. The first smear was obtained within the first or the second week of life (time T1), and the second was taken between the eighth and the eighteenth week of life (time T2), prior to cleft lip and palate repair. All samples were obtained using a sterile EUROTUBO ${ }^{\circledR}$ collection swab 


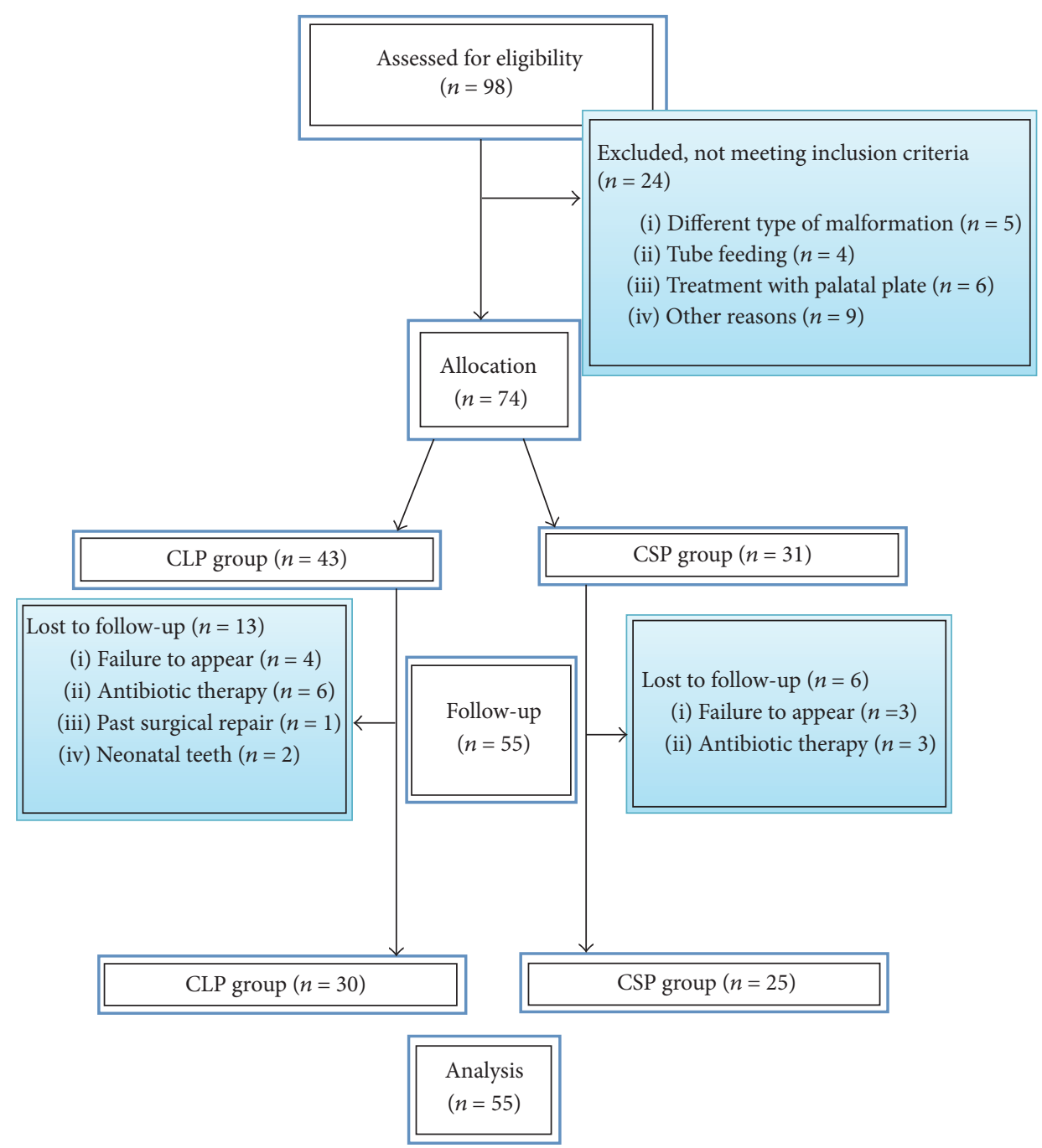

FIGURE 1: Number of subjects recruited and flow of patients within the study.

with Amies transport medium (DELTALAB, Rubi, Spain) and were delivered to the Department of Microbiology and Immunology in Zabrze within $1 \mathrm{~h}$, where the material underwent analysis.

2.2. Microbiological Examination. The samples collected for microbiological investigation were smears from the palatal mucosa and smears from the dorsum of the tongue. All the studied samples were inoculated on a solid culture media from Biomerieux (Marcy l'Etoile, France): Columbia agar with 5\% ram blood, MacConkey agar, Mannitol salt/Chapman agar, and Sabouraud agar. The bacteria were grown on suitable media at $37^{\circ} \mathrm{C}$ in aerobic conditions. Yeast fungi of the Candida species were multiplied on selective solid medium Sabouraud agar at a temperature of $35^{\circ} \mathrm{C}$ in aerobic conditions. Before identification, the studied microorganisms were cultured and isolated on solid nonselective medium Columbia agar with $5 \%$ of ram blood in order to evaluate the morphology of the pure culture, haemolytic activity, and pigmentation production. We also used related selective solid media, such as MacConkey agar for rods and Chapman agar for cocci. After isolation and further culture of each microorganism, their species were identified using the following set of reagents: Slidex Staph Plus, ID Color Catalase, Oxidase Reagent, and Api Candida (Biomerieux, Marcy l'Etoile, France), as well as STAPHYtest 24, STREPTOtest 24, ENTEROtest 24N, and NEFERMtest 24N (Erba-Lachema, Brno, Czech Republic). In the case of Gram positive, catalase negative beta-haemolytic cocci we analysed the presence of group antigens using Slidex Strepto Plus kit, and a sensitivity test to optochin was performed to clearly differentiate pneumococci from other streptococci. Species of microorganisms were identified using conventional methods, with the use of commercial test kits (STAPHYtest 24, STREPTOtest 24, ENTEROtest $24 \mathrm{~N}$, and NEFERMtest $24 \mathrm{~N}$ ), from among the MIKROLATEST identification kits manufactured by Erba-Lachema. The MIKROLATEST kits are a standardized micromethod system for rapid, reliable routine identification of the most clinically important bacteria and yeasts, in every case on the basis of 24 biochemical tests placed in microwells. For evaluation of identification results, we used TNW LITE 6.5 software 
TABLE 1: Descriptive statistics and statistical comparisons between CLP group and CSP group.

\begin{tabular}{|c|c|c|c|}
\hline & CLP group $(n=30)$ & CSP group $(n=25)$ & $p$ value \\
\hline \multicolumn{4}{|l|}{ Age in days, median (IQR) } \\
\hline At $\mathrm{T} 1$ & $7.5(4-11)$ & $7(4-10)$ & $0.931^{\mathrm{a}}$ \\
\hline At $\mathrm{T} 2$ & $77(66-91)$ & $70(63-90)$ & $0.726^{\mathrm{a}}$ \\
\hline \multicolumn{4}{|l|}{ Gender } \\
\hline Female, $n(\%)$ & $11(36)$ & $14(56)$ & $0.297^{\mathrm{b}}$ \\
\hline Male, $n(\%)$ & $19(64)$ & $11(44)$ & $0.137^{\mathrm{b}}$ \\
\hline \multicolumn{4}{|l|}{ Mode of delivery } \\
\hline Natural $n(\%)$ & $13(43)$ & $16(64)$ & $0.139^{\mathrm{b}}$ \\
\hline Caesarean section $n(\%)$ & $23(77)$ & $9(36)$ & $0.038^{b}$ \\
\hline Birth weight (grams), median (IQD) & $3000(2400-3800)$ & $3000(2500-3800)$ & $0.938^{\mathrm{b}}$ \\
\hline
\end{tabular}

${ }^{\mathrm{a}}$ Mann-Whitney $U$ test; ${ }^{\mathrm{b}}$ chi-square test.

The results printed in boldface reached statistical significance $(p<0.05)$.

as recommended by Erba-Lachema. Identification of the microorganism species using these reagents was performed according to the vendors' protocols.

2.3. Data Collection. At T1 and T2, we assessed the prevalence of the given bacterial species (the percentage) found in the oral cavities of subjects with CLP or CSP group. We also analysed the proportions in which the palate $(\mathrm{A} 1, \mathrm{~B} 1)$ and tongue (A2, B2) were colonised by each microorganism.

The intensity of bacterial growth was considered using the following scale: (1) scant growth, (2) medium growth, and (3) abundant growth. The evolution of the microbiota between $\mathrm{T} 1$ and T2 was assessed separately for both groups by analysing the number of patients for whom a given microorganism was detected at both timepoints or only at $\mathrm{T} 1$ or $\mathrm{T} 2$.

2.4. Statistics. Descriptive statistics are expressed as number and percentage and as median and interquartile range, as appropriate. The distributions of continuous variables were compared with a Mann-Whitney $U$ test and proportions with the chi-square test.

The differences in the frequency of the occurrence of each bacterial species between CLP and CSP group at T1 and T2 were assessed using the chi-square test.

The McNemar test was used to compare within-group differences in the frequency of detection of single bacterial species between $\mathrm{T} 1$ and $\mathrm{T} 2$. Odds ratios (ORs) with 95\% confidence intervals (CIs) were computed. All statistics were two-tailed, and the significance level was defined as $p<0.05$. Statistical analyses were performed using Statistica v.10.

\section{Results}

3.1. Gum Pad Stage of the Neonatal Period (T1). The subjects with CLP were delivered by caesarean section significantly more frequently compared to the CSP group $(p=0.038)$. The study groups were age- and birth weight-matched (Table 1).

The genus Streptococcus was found most frequently in both the CLP and CSP groups in the neonatal period (63\%), whereas Streptococcus mitis (S. mitis) was the most frequently observed species $(63.3 \%$ and $60.0 \%$, resp.) (Table 2$)$. Moreover, the frequency of methicillin-sensitive Staphylococcus aureus ( $S$. aureus MSSA) was significantly higher in the CLP group ( $p=0.020)$ than in the CSP group (Table 2 ).

The majority of Streptococcus species showed abundant growth in subjects of both the CLP and CSP groups (Table 2).

A difference in microbiota colonisation between the palate (A1, B1) and tongue (A2, B2) was observed in both groups. This change was related to the bacteria that appeared with frequencies higher than $20 \%$. S. mitis and S. salivarius were dominant on the tongue (A2, B2), whereas Streptococcus sanguinis (S. sanguinis) prevailed on the palate (A1, B1). The remaining bacteria did not demonstrate significant differences in their colonisation of the palate and tongue (Table 2).

3.2. Gum Pad Stage of the Infancy Period (T2). S. salivarius was the most frequently isolated bacterial species in both CLP and CSP patients (100\% and 84\%, resp.; Table 3). Furthermore, compared to the CSP group, subjects from the CLP group presented a significantly higher percentage of the following bacterial species: $S$. mitis $(p=0.002), S$. salivarius $(p=0.022), S$. aureus MSSA $(p<0.001)$, Staphylococcus epidermidis (S. epidermidis) $(p<0.001)$, and the members of the Enterobacteriaceae family, that is, Enterobacter cloacae (E. cloacae) ( $p=0.007)$, Klebsiella pneumoniae (K. pneumoniae) $(p<0.001)$, and Klebsiella oxytoca (K. oxytoca) $(p<0.001)$ (Table 3).

The proportions in which the palate $(\mathrm{A} 1, \mathrm{~B} 1)$ and the tongue (A2, B2) were colonised by each microorganism were similar to that observed in the neonatal period. S. mitis and S. salivarius were dominant on the tongue (A2, B2), whereas S. sanguinis prevailed on the palate (A1, B1) (Table 3 ).

Moreover, infants from CLP group and from CSP group presented with Streptococcus agalactiae (S. agalactiae) (6.6\%, $16 \%)$, and infants with CLP also presented with Streptococcus pyogenes (S. pyogenes) (13.3\%) (Table 3$)$.

\subsection{Development of the Microbiota (between T1 and T2)}

3.3.1. CLP Group. In the CLP group, between $\mathrm{T} 1$ and $\mathrm{T} 2$, a statistically significant increase was observed in the prevalence 
TABLE 2: Statistical comparison of microorganism frequency (prevalence), colonisation, and growth intensity between CLP group and CSP group at $\mathrm{T} 1$.

\begin{tabular}{|c|c|c|c|c|c|c|c|c|c|}
\hline \multirow{3}{*}{ Microorganism } & \multicolumn{4}{|c|}{ CLP group $(n=30)$} & \multirow{3}{*}{$p$ value $^{\mathrm{a}}$} & \multicolumn{4}{|c|}{ CSP group $(n=25)$} \\
\hline & \multirow{2}{*}{$F(\%)$} & \multicolumn{2}{|c|}{ Colonisation (\%) } & \multirow{2}{*}{ GI } & & \multirow{2}{*}{$F(\%)$} & \multicolumn{2}{|c|}{ Colonisation (\%) } & \multirow{2}{*}{ GI } \\
\hline & & $\mathrm{A} 1$ & $\mathrm{~A} 2$ & & & & B1 & $\mathrm{B} 2$ & \\
\hline Streptococcus mitis & 63.3 & 73 & 100 & 3 & 0.458 & 60.0 & 53.3 & 93.3 & 3 \\
\hline Streptococcus oralis & 6.6 & 100 & 100 & 3 & 0.665 & 4.0 & 100 & 0 & 3 \\
\hline Streptococcus pneumoniae & 3.3 & 100 & 100 & 3 & 0.590 & 4.0 & 100 & 100 & 3 \\
\hline Streptococcus sanguinis & 20 & 100 & 50 & 3 & 0.486 & 28.0 & 85.7 & 57.1 & 3 \\
\hline Streptococcus salivarius & 26.6 & 50 & 87.5 & 3 & 0.100 & 48.0 & 41.6 & 66.6 & 3 \\
\hline Streptococcus vestibularis & 10 & 100 & 100 & 3 & 0.090 & 8.0 & 100 & 100 & 3 \\
\hline Streptococcus bovis biovar I & 26.6 & 75 & 87.5 & 3 & 0.343 & 16.0 & 75 & 75 & 3 \\
\hline Streptococcus acidominimus & 6.6 & 50 & 50 & 3 & 0.492 & 12.0 & 100 & 100 & 3 \\
\hline Streptococcus dysgalactiae & 6.6 & 50 & 50 & 2 & 0.848 & 8.0 & 100 & 100 & 3 \\
\hline Streptococcus uberis & 6.6 & 100 & 100 & 3 & 0.663 & 4.0 & 100 & 100 & 3 \\
\hline Streptococcus anginosus & 6.6 & 80 & 100 & 2 & 0.188 & - & - & - & - \\
\hline Streptococcus intermedius & 16.6 & 100 & 50 & 3 & 0.952 & 16.0 & 100 & 50 & 3 \\
\hline Streptococcus constellatus & 6.6 & 100 & 50 & 3 & 0.188 & - & - & - & - \\
\hline Lactobacillus spp. & 13.3 & 100 & 50 & 3 & 0.777 & 16.0 & 75 & 75 & 3 \\
\hline Gemella haemolysans & 16.6 & 100 & 60 & 3 & 0.952 & 16.0 & 50 & 75 & 3 \\
\hline Gemella morbillorum & 20 & 100 & 60 & 3 & 0.076 & 4.0 & 100 & 100 & 3 \\
\hline Enterococcus spp. & 6.6 & 100 & 50 & 3 & 0.665 & 4.0 & 100 & 100 & 1 \\
\hline Staphylococcus aureus MSSA & 40 & 83.3 & 83.3 & 3 & 0.020 & 12.0 & 100 & 75 & 2 \\
\hline Staphylococcus aureus MRSA & 3.3 & 100 & 100 & 2 & 0.359 & - & - & - & - \\
\hline Staphylococcus xylosus & 13.3 & 10 & 50 & 2 & 0.231 & 4.0 & 100 & 100 & 3 \\
\hline Staphylococcus epidermidis & 33.3 & 20 & 23,3 & 2 & 0.833 & 36.0 & 88.8 & 88.8 & 2 \\
\hline Staphylococcus hominis & 10 & 100 & 100 & 1 & 0.103 & - & - & - & - \\
\hline Staphylococcus haemolyticus & 3.3 & 100 & 100 & 3 & 0.773 & 8.0 & 100 & 100 & 3 \\
\hline Staphylococcus lugdunensis & 10 & 100 & 100 & 2 & 0.103 & 8.0 & 100 & 50 & 1 \\
\hline Lactococcus lactis & 3.3 & 100 & 0 & 2 & 0.773 & 8.0 & 0 & 100 & 1 \\
\hline Neisseria spp. & 16.6 & 100 & 80 & 2 & 0.099 & 36.0 & 88,8 & 100 & 2 \\
\hline Moraxella spp. & 3.3 & 100 & 100 & 1 & 0.899 & 4.0 & 100 & 100 & 1 \\
\hline Acinetobacter lwoffii & 6.6 & 100 & 100 & 1 & 0.665 & 4.0 & 0 & 100 & 1 \\
\hline Acinetobacter baumannii & 3.3 & 100 & 100 & 1 & 0.899 & 4.0 & 100 & 100 & 3 \\
\hline Enterobacter cloacae & 10 & 100 & 100 & 2 & 0.870 & 12.0 & 100 & 100 & 1 \\
\hline Enterobacter kobei & 10 & 100 & 100 & 2 & 0.393 & 4.0 & 100 & 100 & 2 \\
\hline Enterobacter aerogenes & 6.6 & 75 & 100 & 2 & 0.841 & 8.0 & 100 & 100 & 2 \\
\hline Enterobacter asburiae & 3.3 & 100 & 100 & 2 & 0.889 & 4.0 & 100 & 100 & 2 \\
\hline Serratia liquefaciens & 6.6 & 100 & 50 & 1 & 0.188 & - & - & - & - \\
\hline Serratia fonticola & 10 & 100 & 33.3 & 2 & 0.465 & 4.0 & 100 & 100 & 1 \\
\hline Klebsiella pneumoniae & 20 & 100 & 100 & 3 & 0.424 & 12.0 & 100 & 100 & 2 \\
\hline Klebsiella oxytoca & 16.6 & 100 & 80 & 3 & 0.494 & 24.0 & 100 & 83,3 & 2 \\
\hline Citrobacter spp. & 3,3 & 100 & 100 & 3 & 0.590 & 4.0 & 100 & 100 & 2 \\
\hline Escherichia coli & 20 & 100 & 100 & 1 & 0.424 & 12.0 & 66.6 & 75 & 2 \\
\hline
\end{tabular}

$F$, frequency, that is, percentage (\%) of subjects from CLP or CSP group with a given microorganism; colonisation, percentage (\%) of smears of a given bacterial species from the palate and/or from the tongue; A1, smears were obtained from palatal mucosa on the cleft margin in CLP subjects; A2, smears were obtained from the dorsum of the tongue in CLP subjects; B1, smears were obtained from the palatal mucosa in CSP subjects; B2, smears were obtained from the dorsum of the tongue in CSP subjects; GI, growth intensity; ${ }^{a}$ chi- square test; results printed in boldface have reached statistical significance $(p<0.05)$.

of 9 bacterial species: $S$. mitis $(p=0.006)$, S. sanguinis $(p=$ $0.012), S$. salivarius $(p<0.001), S$. aureus MSSA $(p<0.001)$, S. epidermidis $(p<0.001)$, Neisseria spp. $(p=0.007)$, $E$. cloacae $(p=0.021)$, K. pneumoniae $(p=0.006)$, and $K$. oxytoca $(p<0.001)$. Moreover, a statistically significant decrease in the percentage of Gemella morbillorum ( $p=0.041$ ) was revealed at T2. The odds ratio for $S$. salivarius in the CLP group during T2 was 22 times higher compared to T1, OR $=22$ [95\% CI, 
TABLE 3: Statistical comparison of microorganism frequency (prevalence), colonisation, and growth intensity between CLP group and CSP group at $\mathrm{T} 2$.

\begin{tabular}{|c|c|c|c|c|c|c|c|c|c|}
\hline \multirow{3}{*}{ Microorganism } & \multicolumn{4}{|c|}{ CLP group $(n=30)$} & \multirow{3}{*}{$p$ value $^{\mathrm{a}}$} & \multicolumn{4}{|c|}{ CSP group $(n=25)$} \\
\hline & \multirow{2}{*}{$F(\%)$} & \multicolumn{2}{|c|}{ Colonisation (\%) } & \multirow{2}{*}{ GI } & & \multirow{2}{*}{$F(\%)$} & \multicolumn{2}{|c|}{ Colonisation (\%) } & \multirow{2}{*}{ GI } \\
\hline & & $\mathrm{A} 1$ & $\mathrm{~A} 2$ & & & & B1 & B2 & \\
\hline Streptococcus mitis & 100 & 60 & 100 & 3 & 0.002 & 56 & 64 & 100 & 3 \\
\hline Streptococcus oralis & 10 & 100 & 100 & 3 & 0.103 & - & - & - & - \\
\hline Streptococcus pneumoniae & 13.3 & 100 & 100 & 3 & 0.777 & 16.0 & 100 & 100 & 3 \\
\hline Streptococcus sanguinis & 50 & 100 & 46.6 & 3 & 0.458 & 40.0 & 100 & 60 & 3 \\
\hline Streptococcus salivarius & 100 & 50 & 100 & 3 & 0.022 & 84.0 & 47.6 & 100 & 3 \\
\hline Streptococcus vestibularis & 10.0 & 66.6 & 100 & 3 & 0.103 & - & - & - & - \\
\hline Streptococcus bovis biovar I & 13.3 & 50 & 50 & 3 & 0.174 & 28.0 & 100 & 85,7 & 3 \\
\hline Streptococcus acidominimus & 13,3 & 50 & 100 & 3 & 0.174 & 28.0 & 100 & 0.0 & 3 \\
\hline Streptococcus agalactiae & 6.6 & 100 & 100 & 3 & 0.264 & 16.0 & 100 & 50 & 3 \\
\hline Streptococcus pyogenes & 13.3 & 100 & 100 & 3 & 0.058 & - & - & - & - \\
\hline Streptococcus uberis & 6.6 & 100 & 100 & 3 & 0.487 & 12.0 & 100 & 100 & 3 \\
\hline Streptococcus anginosus & 13.3 & 50 & 100 & 2 & 0.058 & - & - & - & - \\
\hline Streptococcus intermedius & 26.6 & 100 & 100 & 3 & 0.343 & 16.0 & 100 & 85.7 & 3 \\
\hline Lactobacillus spp. & 13.3 & 75 & 100 & 3 & 0.058 & - & - & - & - \\
\hline Gemella haemolysans & 26.6 & 62.5 & 100 & 2 & 0.177 & 12.0 & 100 & 100 & 3 \\
\hline Enterococcus spp. & 13.3 & 75.0 & 100 & 3 & 0.885 & 12.0 & 100 & 66.6 & 3 \\
\hline Staphylococcus aureus MSSA & 93.3 & 71.4 & 64.2 & 3 & $<0.001$ & 20.0 & 80 & 100 & 3 \\
\hline Staphylococcus epidermidis & 83.3 & 92.0 & 100 & 2 & $<0.001$ & 28.0 & 71.4 & 57.1 & 3 \\
\hline Staphylococcus hominis & 13.3 & 100 & 100 & 1 & 0.058 & - & - & - & - \\
\hline Lactococcus lactis & 13.3 & 100 & 0.0 & 3 & 0.058 & - & - & - & - \\
\hline Neisseria spp. & 53.3 & 56.2 & 93.7 & 3 & 0.695 & 48.0 & 83.3 & 66.6 & 3 \\
\hline Enterobacter cloacae & 36.6 & 90.9 & 100 & 2 & 0.007 & - & - & - & - \\
\hline Enterobacter kobei & 26.6 & 100 & 100 & 3 & 0.053 & - & - & - & - \\
\hline Enterobacter aerogenes & 13.3 & 50 & 75 & 2 & 0.058 & - & - & - & - \\
\hline Hafnia alvei & 3.3 & 100 & 100 & 2 & 0.359 & - & - & - & - \\
\hline Klebsiella pneumoniae & 53.3 & 87.5 & 100 & 3 & $<0.001$ & - & - & - & - \\
\hline Klebsiella oxytoca & 76.6 & 43.4 & 56.5 & 3 & $<0.001$ & - & - & - & - \\
\hline Escherichia coli & 36.6 & 100 & 100 & 2 & 0.311 & 24.0 & 100 & 83.3 & 2 \\
\hline Candida albicans & 6.6 & 100 & 100 & 2 & 0.190 & - & - & - & - \\
\hline
\end{tabular}

$F$, frequency, that is, percentage (\%) of subjects from CLP group or CSP group with a given microorganism; colonisation, percentage (\%) of smears of a given bacterial species from the palate and/or from the tongue; A1, smears were obtained from palatal mucosa on the cleft margin in CLP subjects; A2, smears were obtained from the dorsum of the tongue in CLP subjects; B1, smears were obtained from the palatal mucosa in CSP subjects; B2, smears were obtained from the dorsum of the tongue in CSP subjects; GI, growth intensity; ${ }^{a}$ chi-square test; results printed in boldface have reached statistical significance $(p<0.05)$.

2.96-16.21]. The odds ratio for $S$. aureus MSSA, OR $=16$ [95\% CI, 2.12-12.65], and K. oxytoca, OR = 18 [95\% CI, 2.40-13.83], were 16 and 18 times higher, respectively, at $\mathrm{T} 2$ than at $\mathrm{T} 1$ (Table 4).

3.3.2. CSP Group. A statistically significant increase in the frequency of $S$. salivarius was observed $(p=0.022)$ at T2, OR $=5.5[95 \% \mathrm{CI}, 1.219-24.814]$. The frequency of the occurrence of the remaining bacteria changed insignificantly (Table 5).

\section{Discussion}

The study presents the prevalence of oral microbiota in several-day old newborns with CLP and CSP and changes in microbial population during the infant predental period prior to surgical procedure, which has not previously been described in the literature.

The prevalence of nonpathogenic commensal oral bacteria (i.e., S. mitis and S. salivarius) was revealed in T1 in subjects from both CLP and CSP groups. Long and Swenson confirmed the ability of $S$. mitis and $S$. salivarius to adhere to oral epithelial cells in the oral cavity epithelia of 1-day-old newborns [22]. The early colonisation of the oral cavity by streptococci facilitates further colonisation by other strains and plays a crucial role in maintaining a healthy oral cavity throughout life $[5,22]$. Thus, mechanisms exist to enable physiological colonisation of the mucous membrane by nonpathogenic microbiota in both CLP and CSP group patients during the neonatal period despite different local conditions related to the occurrence of cleft. An interesting finding of this 
TABLE 4: Development of the oral microbiota between T1 and T2 in CLP subjects.

\begin{tabular}{|c|c|c|c|c|c|c|c|}
\hline Microorganism & $\begin{array}{c}{ }^{\dagger}+\rightarrow+ \\
n(\%)\end{array}$ & $\begin{array}{c}{ }^{\ddagger}+\rightarrow- \\
n(\%)\end{array}$ & $\begin{array}{c}\mathbb{S}_{-\rightarrow+} \rightarrow \\
n(\%)\end{array}$ & $\begin{array}{c}{ }^{\#}-\rightarrow- \\
n(\%)\end{array}$ & $p$ value $^{\mathrm{a}}$ & OR & $95 \% \mathrm{CI}$ \\
\hline Streptococcus mitis & $19(63)$ & 0 & $11(37)$ & 0 & 0.006 & NA & NA \\
\hline Streptococcus oralis & $1(3)$ & 0 & $2(6)$ & $27(90)$ & 0.479 & NA & NA \\
\hline Streptococcus pneumoniae & $1(3)$ & 0 & $3(10)$ & $21(70)$ & 0.248 & NA & NA \\
\hline Streptococcus sanguinis & $5(16)$ & $1(3)$ & $10(33)$ & $14(47)$ & 0.012 & 10 & $1.28-78.12$ \\
\hline Streptococcus salivarius & $7(23)$ & $1(3)$ & $22(73)$ & 0 & $<0.001$ & 22 & $2.96-16.21$ \\
\hline Streptococcus vestibularis & $2(6)$ & $1(3)$ & $1(3)$ & $26(87)$ & 0.497 & 2 & $0.18-22.05$ \\
\hline Streptococcus bovis biovar I & $2(6)$ & $6(20)$ & $2(6)$ & $20(67)$ & 0.288 & 3 & $0.72-49.83$ \\
\hline Streptococcus acidominimus & $2(6)$ & 0 & $2(6)$ & $26(87)$ & 0.497 & NA & NA \\
\hline Streptococcus agalactiae & 0 & 0 & $2(6)$ & $28(93)$ & 0.497 & NA & NA \\
\hline Streptococcus pyogenes & 0 & 0 & $4(13)$ & $26(87)$ & 0.125 & NA & NA \\
\hline Streptococcusdysgalactiae & 0 & $2(6)$ & 0 & $28(93)$ & 0.497 & NA & NA \\
\hline Streptococcus uberis & $1(3)$ & $1(3)$ & $1(3)$ & $27(90)$ & 1.00 & 1 & $0.06-15.98$ \\
\hline Streptococcus anginosus & $1(3)$ & $1(3)$ & $2(6)$ & $26(87)$ & 1.00 & 2 & $0.18-22.05$ \\
\hline Streptococcus intermedius & $5(17)$ & 0 & $3(10)$ & $22(73)$ & 0.248 & NA & NA \\
\hline Streptococcus constellatus & $3(10)$ & $1(3)$ & $1(3)$ & $25(83)$ & 1.00 & 1 & $0.06-15.98$ \\
\hline Lactobacillus spp. & $3(10)$ & $1(3)$ & $5(17)$ & $21(70)$ & 0.218 & 5 & $0.58-42.79$ \\
\hline Gemella haemolysans & $5(17)$ & 0 & $3(10)$ & $22(73)$ & 0.248 & NA & NA \\
\hline Gemella morbillorum & 0 & $6(20)$ & 0 & $24(80)$ & 0.041 & NA & NA \\
\hline Enterococcus spp. & $2(6)$ & 0 & $2(6)$ & $26(87)$ & 0.497 & NA & NA \\
\hline Staphylococcus aureus MSSA & $12(40)$ & $1(3)$ & $16(53)$ & $1(3)$ & $<0.001$ & 16 & $2.12-12.65$ \\
\hline Staphylococcus aureus MRSA & 0 & $1(3)$ & 0 & $29(97)$ & 1 & NA & NA \\
\hline Staphylococcus xylosus & 0 & $4(13)$ & $4(13)$ & $26(87)$ & 0.733 & 1 & $0.25-3.99$ \\
\hline Staphylococcus epidermidis & $9(30)$ & $1(3)$ & $16(53)$ & $4(13)$ & $<0.001$ & 16 & $2.12-12.65$ \\
\hline Staphylococcus hominis & $1(3)$ & $2(6)$ & $3(10)$ & $24(80)$ & 1 & 1.5 & $0.25-8.97$ \\
\hline Staphylococcus haemolyticus & 0 & $1(3)$ & 0 & $29(97)$ & 1 & NA & NA \\
\hline Staphylococcus lugdunensis & 0 & $3(10)$ & 0 & $27(90)$ & 0.248 & NA & NA \\
\hline Lactococcus lactis & $1(3)$ & 0 & $3(10)$ & $26(87)$ & 0.248 & NA & NA \\
\hline Neisseria spp. & $3(10)$ & $2(6)$ & $13(43)$ & $12(40)$ & 0.007 & 6.5 & $1.46-28.80$ \\
\hline Moraxella spp. & 0 & $1(3)$ & 0 & $29(97)$ & 1 & NA & NA \\
\hline Acinetobacter lwoffii & 0 & $2(6)$ & 0 & $28(93)$ & 0.479 & NA & NA \\
\hline Acinetobacter baumannii & 0 & $1(3)$ & 0 & $29(97)$ & 1 & NA & NA \\
\hline Enterobacter cloacae & $3(10)$ & 0 & $9(30)$ & $18(60)$ & 0.021 & NA & NA \\
\hline Enterobacter kobei & $2(6)$ & $1(3)$ & $6(20)$ & $21(70)$ & 0.125 & 6 & $0.72-49.83$ \\
\hline Enterobacter aerogenes & $2(6)$ & 0 & $2(6)$ & $26(87)$ & 0.479 & NA & NA \\
\hline Enterobacter asburiae & 0 & $1(3)$ & 0 & $29(97)$ & 1 & NA & NA \\
\hline Serratia liquefaciens & 0 & $2(6)$ & 0 & $28(93)$ & 0.479 & NA & NA \\
\hline Serratia fonticola & 0 & $3(10)$ & 0 & $27(90)$ & 0.248 & NA & NA \\
\hline Klebsiella pneumoniae & $5(17)$ & $1(3)$ & $11(37)$ & $13(43)$ & 0.006 & 11 & $1.42-85.20$ \\
\hline Klebsiella oxytoca & $4(13)$ & $1(3)$ & $18(60)$ & $7(23)$ & $<0.001$ & 18 & $2.40-13.83$ \\
\hline Citrobacter spp. & 0 & $1(3)$ & 0 & $29(97)$ & 1 & NA & NA \\
\hline Escherichia coli & $1(3)$ & $5(17)$ & $10(33)$ & $14(47)$ & 0.301 & 2 & $0.68-5.85$ \\
\hline
\end{tabular}

${ }^{\dagger}$ Number $(n)$ and percentage (\%) of patients with the bacterial species present at both $\mathrm{T} 1$ and $\mathrm{T} 2$.

* Number $(n)$ and percentage (\%) of patients with the bacterial species present at T1 but not at T2;

${ }^{\S}$ Number $(n)$ and percentage (\%) of patients without the bacterial species present at T1 but with it at T2.

${ }^{\#}$ Number $(n)$ and percentage (\%) of patients without the bacterial species present at T1 or T2.

${ }^{a} \mathrm{McNemar}$ Test; OR, odds ratio; 95\% CI, confidence interval of 95\%; NA, not applicable.

Results in boldface have reached statistical significance $(p<0.05)$. 
TABLE 5: Development of the oral microbiota between T1 and T2 in CSP subjects.

\begin{tabular}{|c|c|c|c|c|c|c|c|}
\hline Microorganism & $\begin{array}{l}{ }^{\dagger}+\rightarrow+ \\
n(\%)\end{array}$ & $\begin{array}{c}{ }^{\ddagger}+\rightarrow- \\
n(\%)\end{array}$ & $\begin{array}{c}{ }^{\S}-\rightarrow+ \\
n(\%)\end{array}$ & $\begin{array}{c}{ }_{-}^{*} \rightarrow- \\
n(\%)\end{array}$ & $p$ value $^{\mathrm{a}}$ & OR & $95 \% \mathrm{CI}$ \\
\hline Streptococcus mitis & $13(52)$ & $2(8)$ & $1(4)$ & $9(36)$ & 1.00 & NA & $\mathrm{NA}$ \\
\hline Streptococcus oralis & 0 & $1(4)$ & 0 & $24(96)$ & 1.00 & NA & $\mathrm{NA}$ \\
\hline Streptococcus pneumoniae & $1(4)$ & 0 & $3(12)$ & $21(84)$ & 0.248 & NA & NA \\
\hline Streptococcus sanguinis & $4(16)$ & $3(12)$ & $6(24)$ & $12(48)$ & 0.507 & 2 & $0.50-7.99$ \\
\hline Streptococcus salivarius & $10(40)$ & $2(8)$ & $11(44)$ & $2(8)$ & 0.022 & 5.5 & $1.21-24.81$ \\
\hline Streptococcus vestibularis & 0 & $2(8)$ & 0 & $23(92)$ & 0.479 & NA & NA \\
\hline Streptococcus bovis biovar I & $1(4)$ & $3(12)$ & $6(24)$ & $15(60)$ & 0.507 & 2 & $0.50-7.99$ \\
\hline Streptococcus acidominimus & $1(4)$ & $2(8)$ & $6(24)$ & $16(64)$ & 0.289 & 3 & $0.60-14.86$ \\
\hline Streptococcus agalactiae & 0 & 0 & $4(16)$ & $21(84)$ & 0.133 & NA & NA \\
\hline Streptococcus pyogenes & 0 & 0 & 0 & 0 & 1.00 & NA & $\mathrm{NA}$ \\
\hline Streptococcus dysgalactiae & 0 & $2(8)$ & 0 & $23(92)$ & 0.479 & NA & NA \\
\hline Streptococcus uberis & 0 & $1(4)$ & $3(12)$ & $21(84)$ & 0.617 & 3 & $0.31-28.84$ \\
\hline Streptococcus anginosus & 0 & 0 & 0 & 0 & 1.00 & $\mathrm{NA}$ & NA \\
\hline Streptococcus intermedius & $2(8)$ & $2(8)$ & $2(8)$ & $19(76)$ & 0.617 & 1 & $0.14-7.09$ \\
\hline Streptococcus constellatus & 0 & 0 & 0 & 0 & 1.00 & NA & NA \\
\hline Lactobacillus spp. & 0 & $4(16)$ & $3(12)$ & $18(72)$ & 0.723 & 1.333 & $0.29-5.95$ \\
\hline Gemella haemolysans & $3(12)$ & $1(4)$ & 0 & $21(84)$ & 1.00 & NA & NA \\
\hline Gemella morbillorum & 0 & $4(16)$ & 0 & $21(84)$ & 0.125 & NA & NA \\
\hline Enterococcus spp. & 0 & $1(4)$ & $3(12)$ & $21(84)$ & 0.627 & 3 & $0.31-28.84$ \\
\hline Staphylococcus aureus MSSA & $1(4)$ & $2(8)$ & $4(16)$ & $18(72)$ & 0.687 & 2 & $0.36-10.91$ \\
\hline Staphylococcus xylosus & 0 & $1(4)$ & 0 & $24(96)$ & 1.00 & NA & NA \\
\hline Staphylococcus epidermidis & $5(20)$ & $4(16)$ & $2(8)$ & $14(56)$ & 0.687 & 2 & $0.36-10.91$ \\
\hline Staphylococcus hominis & 0 & 0 & 0 & 0 & 1.00 & NA & NA \\
\hline Staphylococcus haemolyticus & 0 & $2(8)$ & 0 & $23(92)$ & 0.479 & NA & NA \\
\hline Staphylococcus lugdunensis & 0 & $3(12)$ & 0 & $22(88)$ & 0.248 & NA & $\mathrm{NA}$ \\
\hline Lactococcus lactis & 0 & $1(4)$ & $2(8)$ & $22(88)$ & 1.00 & 2 & $0.18-22.05$ \\
\hline Neisseria spp. & $9(36)$ & 0 & $3(12)$ & $13(52)$ & 0.248 & NA & NA \\
\hline Moraxella spp. & 0 & $1(4)$ & 0 & $24(96)$ & 1.00 & NA & $\mathrm{NA}$ \\
\hline Acinetobacter lwoffii & 0 & 0 & 0 & 0 & 1.00 & NA & $\mathrm{NA}$ \\
\hline Acinetobacter baumannii & 0 & 0 & 0 & 0 & 1.00 & NA & NA \\
\hline Enterobacter cloacae & 0 & $3(12)$ & 0 & $22(88)$ & 0.248 & NA & NA \\
\hline Enterobacter kobei & 0 & 0 & 0 & 0 & 1.00 & NA & NA \\
\hline Enterobacter aerogenes & 0 & $2(8)$ & 0 & $23(88)$ & 0.479 & NA & NA \\
\hline Enterobacter asburiae & 0 & 0 & 0 & 0 & 1.00 & NA & NA \\
\hline Hafnia alvei & 0 & $1(4)$ & 0 & $24(96)$ & 1.00 & NA & NA \\
\hline Serratia liquefaciens & 0 & 0 & 0 & 0 & 1.00 & NA & NA \\
\hline Serratia fonticola & 0 & 0 & 0 & 0 & 1.00 & NA & NA \\
\hline Klebsiella pneumoniae & 0 & $12(48)$ & 0 & $13(52)$ & 1.00 & NA & $\mathrm{NA}$ \\
\hline Klebsiella oxytoca & 0 & $6(24)$ & 0 & 19 & 0.312 & NA & NA \\
\hline Citrobacter spp. & 0 & $1(4)$ & 0 & $24(96)$ & 1.00 & NA & NA \\
\hline Escherichia coli & $1(4)$ & $2(8)$ & $5(20)$ & $17(68)$ & 0.453 & 2.5 & $0.48-12.88$ \\
\hline Candida albicans & 0 & $1(4)$ & 0 & $24(96)$ & 1.00 & $\mathrm{NA}$ & NA \\
\hline
\end{tabular}

${ }^{\dagger}$ Number $(n)$ and percentage (\%) of patients with the bacterial species present at both T1 and T2.

${ }^{\ddagger}$ Number $(n)$ and percentage (\%) of patients with the bacterial species present at T1 but not at T2.

${ }^{\S}$ Number $(n)$ and percentage (\%) of patients without the bacterial species present at T1 but with it at $\mathrm{T} 2$.

${ }^{\#}$ Number $(n)$ and percentage (\%) of patients without the bacterial species present at T1 or T2.

${ }^{a}$ McNemar Test; OR, odds ratio; 95\% CI, confidence interval of 95\%; NA, not applicable.

Results in boldface have reached statistical significance $(p<0.05)$. 
study is the demonstration of the occurrence of S. sanguinis in tongue and palate swabs of toothless infants with CSP and CLP during T1 and T2. Arief et al., when analysing the saliva of 3-39-month-old patients with CLP, did not find $S$. sanguinis, either in the preoperative or postoperative period [21]. On the other hand, Caufield et al., in their long-term studies of saliva samples and dental plaque from infants, demonstrated that $S$. sanguinis precedes $S$. mutans colonisations and that both compete for niches on the tooth surface [23]. Other studies also show that colonisation of both species depends on tooth emergence [24, 25]. However, some authors prove that $S$. sanguinis [26] and S. mutans [27] may colonise the oral mucosa of predental infants and the dorsum of the tongue, which is an important ecological niche $[28,29]$. $S$. sanguinis is considered to be the antagonist of $S$. mutans, and early colonisation with $S$. sanguinis delays colonisation by $S$. mutans, which is considered to be a significant factor in the development of caries [23, 30]. Caufield et al. raise the question of whether this phenomenon should be used in the prevention of caries, inducing early colonisation by $S$. sanguinis, and thus delaying colonisation by S. mutans [23].

The distribution of Streptococcus species in the oral cavities of both CLP and CSP subjects demonstrated differences between the tongue and palate. The majority of $S$. salivarius and $S$. mitis strains were cultured from samples collected from the tongue (A2, B2), whereas $S$. sanguinis mainly derived from palate samples (A1, B1). This observation is consistent with the reports of other authors who confirmed a selective ability in the adherence of streptococci to oral epithelial cells $[12,31]$. The majority of streptococci collected from the palate and the tongue showed abundant growth in both CLP and CSP group.

Group A $\beta$-haemolytic streptococci were not found in CLP or CSP group subjects in the neonatal period. However, other potentially virulent pyogenic streptococci were observed, including Streptococcus pneumoniae (S. pneumoniae), Streptococcus dysgalactiae (S. dysgalactiae), and Streptococcus intermedius (S. intermedius). Subjects with CLP presented with all members of the Streptococcus anginosus group (i.e., S. anginosus, S. constellatus, and S. intermedius). These strains can cause acute infections, particularly in immunodeficient individuals. These inflections include brain, mouth, or liver abscesses and endocarditis, whereas S. agalactiae can cause bacteraemia in neonates, acute pulmonary insufficiency, and cerebrospinal meningitis [20]. In a study of the microbiota in patients with lip cleft prior to surgical intervention, Cocco et al. did not detect the presence of group A $\beta$-haemolytic streptococci in any of the patients. However, the researchers observed the species in only $2.3 \%$ of patients with cleft palate [32]. In contrast, Chuo and Timmons detected $\beta$-haemolytic streptococci in $11 \%$ of positive smears taken from patients with cleft lip and/or palate prior to surgical repair [18]. The presence of $\beta$-haemolytic streptococci in the oral cavity of subjects with CLP is related to postoperative complications, such as slow wound healing or the development of abscesses and fistulae [32].

S. aureus was detected in $40 \%$ of CLP subjects and in only $12 \%$ of CSP group subjects in the neonatal period. The difference between the study groups was statistically significant. The variation in the frequency of $S$. aureus between both groups may be related to the type of cleft and different local environmental conditions. In patients with CSP, the oral and nasal cavities form two nearly separate environments, as only the distal part of the soft palate is cleft. A different morphology is found in CLP patients, in whom the oral and nasal cavities are connected, which facilitates communication, including the transmission of mucus, food, saliva, air, and microbiota. In a study of CLP with oronasal fistulae, Tuna et al. emphasised the significance of $S$. aureus transmission from the oral to the nasal cavity in the risk of infection after the surgical treatment of patients with this malformation [19]. A slightly different opinion was expressed by Cocco et al., who questioned the pathogenicity of $S$. aureus in infants under 1 year of age [32]. Similarly, Jolleys and Savage did not observe an increased number of postoperative complications in patients with S.aureus detected in the preoperative period [33].

In both groups of patients, nonpathogenic streptococci were the most prevalent at T2, in the gum pad stage of the infancy period. Among nonpathogenic streptococci, $S$. salivarius and $S$. mitis were the most frequent. S. aureus was the most frequently detected potentially pathogenic strain and was observed in $93.3 \%$ of subjects with CLP and significantly less frequently in subjects with CSP (20\%).

The formation of the microbiota in subjects with CLP proceeded differently than in CSP group subjects. An increased frequency of potential pathogens, mainly $S$. aureus and S. epidermidis, was observed in the CLP group. The odds ratio for these bacteria increased 16 times with development. These results may be explained by the altered anatomical conditions of the oral cavity, which disturbs self-cleaning and the flow of saliva, facilitating the retention of food in the recesses of the cleft and the nasal cavity and changing the physiological exposure of this area to oxygen and carbon dioxide [34].

The significant increase in the members of the family Enterobacteriaceae observed in subjects with CLP (i.e., E. cloacae, K. pneumoniae, and K. oxytoca) may be related to the transmission of this microbiota from the external environment to the oral cavity. The frequent contact of parents' hands with the mucous membranes of the cleft lip, alveolar ridge, and incisive bone during hygiene procedures in the area of the cleft likely plays an important role in this process. The lip massage recommended by orthodontists as part of preoperative preparations may also significantly contribute to the observed changes in microbiota. Similarly, Cocco et al. indicated a high percentage of CLP subjects with Gram-negative organisms isolated preoperatively [32].

The development of the microbiota in CSP subjects was characterised by a statistically significant increase in the prevalence of $S$. salivarius, whereas the frequency of bacteria from the Enterobacteriaceae family decreased insignificantly. Therefore, the formation of the oral microbiota in subjects with CSP shows a tendency similar to that of healthy infants, in whom the number of the environmental Gram-negative rods decreases with age [5].

In conclusion, this study shows that (1) the development of the microbiota in subjects with CLP is accompanied by a 
significant increase in commensal and potentially pathogenic organisms (S. aureus, S. epidermidis, Neisseria spp., K. pneumoniae, and K. oxytoca); (2) S. aureus was detected in neonates with CLP significantly more frequently than in subjects without CLP. The prevalence of $S$. aureus increases significantly with the development of the child, and the odds ratio increases 16 -fold. Patients with CLP are potentially at an increased risk of developing oral infectious diseases. Early oral health maintenance program in patients with CLP should be reinforced.

Further research on early oral microbiota of patients with oral clefts and its effect on later infectious diseases of the oral cavity, especially dental caries and periodontal diseases, is needed.

\section{Conflicts of Interest}

All participating authors of this study have no conflicts of interest.

\section{Acknowledgments}

The authors are grateful to Professor Wojciech Król, who initiated the project, enabled conducting microbiological tests, and had substantial contributions to the conception of the study. This work was supported by Medical University of Silesia in Katowice, academic project (KNW-1-106/K/4/0).

\section{References}

[1] W. Nolte, "Physiology and growth of microorganisms," in Oral Microbiology with Basic Microbiology and Immunology, pp. 2528, The CV Mosby Company, St. Louis, Mo, USA, 3rd edition, 1977.

[2] V. O. Rotimi and B. I. Duerden, "The development of the bacterial flora in normal neonates," Journal of Medical Microbiology, vol. 14, no. 1, pp. 51-62, 1981.

[3] P. L. Holgerson, N. R. Vestman, R. Claesson et al., "Oral microbial profile discriminates breast-fed from formula-fed infants," Journal of Pediatric Gastroenterology and Nutrition, vol. 56, no. 2, pp. 127-136, 2013.

[4] E. Könönen, "Anaerobes in the upper respiratory tract in infancy," Anaerobe, vol. 11, no. 3, pp. 131-136, 2005.

[5] E. Könönen, "Development of oral bacterial flora in young children," Annals of Medicine, vol. 32, no. 2, pp. 107-112, 2000.

[6] E. Kononen, A. Kanervo, A. Takala, S. Asikainen, and H. Jousimies-Somer, "Establishment of oral anaerobes during the first year of life," Journal of Dental Research, vol. 78, no. 10, pp. 1634-1639, 1999.

[7] P. Lif Holgerson, L. Harnevik, O. Hernell, A. C. R. Tanner, and I. Johansson, "Mode of birth delivery affects oral microbiota in infants," Journal of Dental Research, vol. 90, no. 10, pp. 1183-1188, 2011.

[8] S. S. Socransky and S. D. Manganiello, "The oral microbiota of man from birth to senility," Journal of Periodontology, vol. 42, no. 8, pp. 485-496, 1971.

[9] M. Nelun Barfod, K. Magnusson, M. O. Lexner, S. Blomqvist, G. Dahlén, and S. Twetman, "Oral microflora in infants delivered vaginally and by caesarean section," International Journal of Paediatric Dentistry, vol. 21, no. 6, pp. 401-406, 2011.
[10] M.-M. Grönlund, O.-P. Lehtonen, E. Eerola, and P. Kero, "Fecal microflora in healthy infants born by different methods of delivery: permanent changes in intestinal flora after cesarean delivery," Journal of Pediatric Gastroenterology and Nutrition, vol. 28, no. 1, pp. 19-25, 1999.

[11] P. D. Marsh, "Are dental diseases examples of ecological catastrophes?” Microbiology, vol. 149, no. 2, pp. 279-294, 2003.

[12] J. A. Aas, B. J. Paster, L. N. Stokes, I. Olsen, and F. E. Dewhirst, "Defining the normal bacterial flora of the oral cavity," Journal of Clinical Microbiology, vol. 43, no. 11, pp. 5721-5732, 2005.

[13] B. Costa, J. E. de Oliveira Lima, M. R. Gomide, and O. P. da Silva Rosa, "Clinical and microbiological evaluation of the periodontal status of children with unilateral complete cleft lip and palate," The Cleft Palate-Craniofacial Journal, vol. 40, no. 6, pp. 585-589, 2003.

[14] L. L. Cheng, S. L. Moor, and C. T. C. Ho, "Predisposing factors to dental caries in children with cleft lip and palate: a review and strategies for early prevention," Cleft Palate-Craniofacial Journal, vol. 44, no. 1, pp. 67-72, 2007.

[15] S. K. Clarren, B. Anderson, and L. S. Wolf, "Feeding infants with cleft lip, cleft palate, or cleft lip and palate," Cleft Palate Journal, vol. 24, no. 3, pp. 244-249, 1987.

[16] L. H. Meskin, R. J. Gorlin, and R. J. Isaacson, "Abnormal morphology of the soft palate: I. The prevalence of cleft uvula," The Cleft Palate Journal, vol. 35, pp. 342-346, 1964.

[17] H. Perdikogianni, W. Papaioannou, M. Nakou, C. Oulis, and L. Papagiannoulis, "Periodontal and microbiological parameters in children and adolescents with cleft lip and/or palate," International Journal of Paediatric Dentistry, vol. 19, no. 6, pp. 455-467, 2009.

[18] C. B. Chuo and M. J. Timmons, "The bacteriology of children before primary cleft lip and palate surgery," The Cleft PalateCraniofacial Journal, vol. 42, no. 3, pp. 272-276, 2005.

[19] E. B. Tuna, N. Topçuoglu, B. Ilhan, K. Gençay, and G. Kulekçi, "Staphylococcus aureus transmission through oronasal fistula in children with cleft lip and palate," Cleft Palate-Craniofacial Journal, vol. 45, no. 5, pp. 477-480, 2008.

[20] H. P. Mÿburgh and K.-W. Bütow, "Cleft soft palate reconstruction: prospective study on infection and antibiotics," International Journal of Oral and Maxillofacial Surgery, vol. 38, no. 9, pp. 928-932, 2009.

[21] E. M. Arief, Z. Mohamed, and F. M. Idris, "Study of viridans streptococci and Staphylococcus species in cleft lip and palate patients before and after surgery," The Cleft Palate-Craniofacial Journal, vol. 42, no. 3, pp. 277-279, 2005.

[22] S. S. Long and R. M. Swenson, "Determinants of the developing oral flora in normal newborns," Applied and Environmental Microbiology, vol. 32, no. 4, pp. 494-497, 1976.

[23] P. W. Caufield, A. P. Dasanayake, Y. Li, Y. Pan, J. Hsu, and J. M. Hardin, "Natural history of Streptococcus sanguinis in the oral cavity of infants: evidence for a discrete window of infectivity," Infection and Immunity, vol. 68, no. 7, pp. 4018-4023, 2000.

[24] J. Carlsson, H. Grahnén, G. Jonsson, and S. Wikner, "Establishment of Streptococcus sanguis in the mouths of infants," Archives of Oral Biology, vol. 15, no. 12, pp. 1143-1148, 1970.

[25] P. W. Caufield, G. R. Cutter, and A. P. Dasanayake, "Initial acquisition of mutans streptococci by infants: evidence for a discrete window of infectivity," Journal of Dental Research, vol. 72, no. 1, pp. 37-45, 1993.

[26] C. Pearce, G. H. Bowden, M. Evans et al., "Identification of pioneer viridans streptococci in the oral cavity of human 
neonates," Journal of Medical Microbiology, vol. 42, no. 1, pp. 6772, 1995.

[27] A. K. L. Wan, W. K. Seow, L. J. Walsh, P. Bird, D. I. Tudehope, and D. M. Purdie, "Association of streptococcus mutans infection and oral developmental nodules in pre-dentate infants," Journal of Dental Research, vol. 80, no. 10, pp. 1945-1948, 2001.

[28] A. C. R. Tanner, P. M. Milgrom, R. Kent Jr. et al., “The microbiota of young children from tooth and tongue samples," Journal of Dental Research, vol. 81, no. 1, pp. 53-57, 2002.

[29] R. J. Berkowitz, "Acquisition and transmission of mutans streptococci," Journal of the California Dental Association, vol. 31, no. 2, pp. 135-138, 2003.

[30] S. Alaluusua and O. Renkonen, "Streptococcus mutans establishment and dental caries experience in children from 2 to 4 years old," European Journal of Oral Sciences, vol. 91, no. 6, pp. 453-457, 1983.

[31] R. J. Gibbons and J. van Houte, "Selective bacterial adherence to oral epithelial surfaces and its role as an ecological determinant," Infection and Immunity, vol. 3, no. 4, pp. 567-573, 1971.

[32] J. F. Cocco, J. W. Antonetti, J. L. Burns, J. P. Heggers, and S. J. Blackwell, "Characterization of the nasal, sublingual, and oropharyngeal mucosa microbiota in cleft lip and palate individuals before and after surgical repair," The Cleft PalateCraniofacial Journal, vol. 47, no. 2, pp. 151-155, 2010.

[33] A. Jolleys and J. P. Savage, "Healing defects in cleft palate surgery - the role of infection," British Journal of Plastic Surgery, vol. 16, pp. 134-139, 1963.

[34] A. Machorowska-Pieniążek, T. Morawiec, A. Mertas, M. Tanasiewicz, A. Dziedzic, and W. Król, "Influence of propolis on hygiene, gingival condition, and oral microflora in patients with cleft lip and palate treated with fixed orthodontic appliances," Evidence-Based Complementary and Alternative Medicine, vol. 2013, Article ID 183915, 2013. 

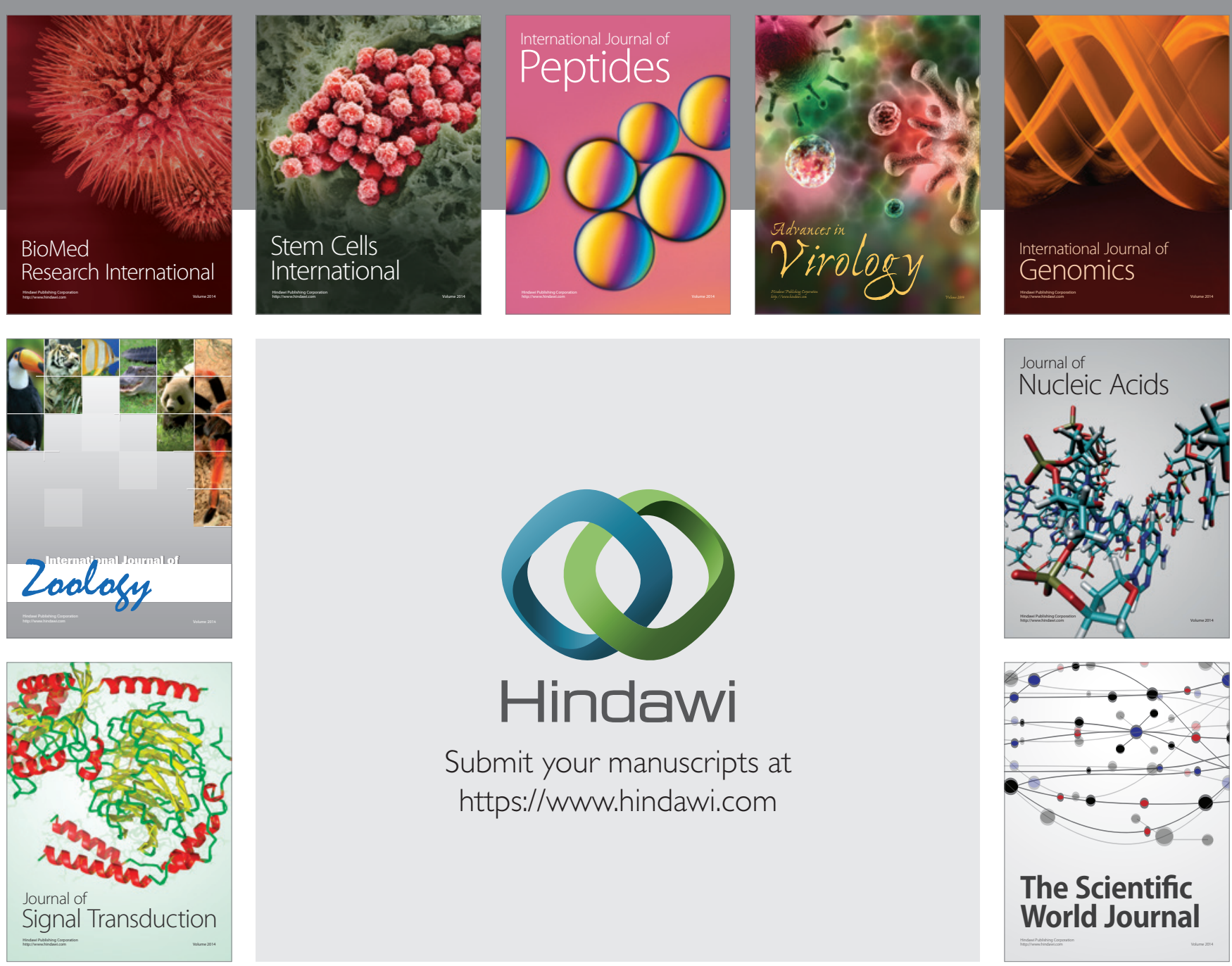

Submit your manuscripts at

https://www.hindawi.com
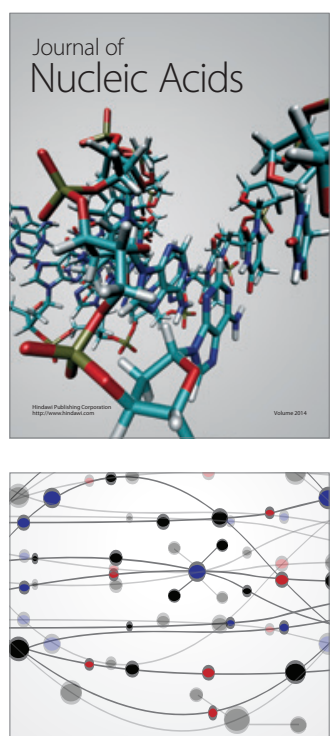

The Scientific World Journal
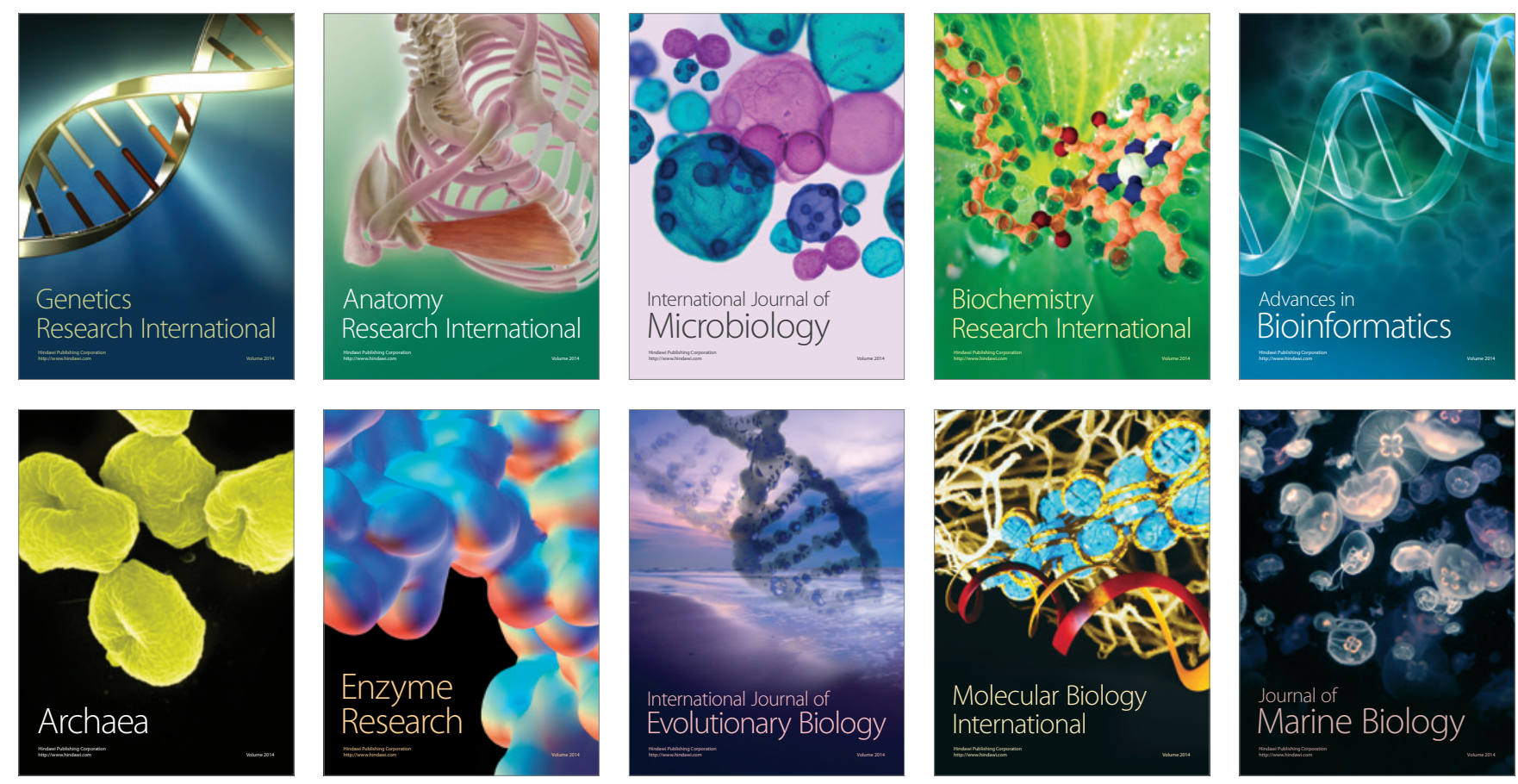